\title{
Acute psychosis in pregnancy - A case managed effectively in accordance with the MBRRACE recommendations
}

\author{
Eisha Jain ${ }^{1}$, Vahini Tharmakulasingham ${ }^{1}$ and Radhika Viswanatha ${ }^{2 *}$ \\ ${ }^{1}$ St George's University of London, UK \\ ${ }^{2}$ Obstetric and Gynaeoclogy Consultant, Epsom and St Helier University Hospitals NHS Trust, UK
}

\section{Introduction}

Women are at a higher risk of experiencing new-onset severe mental illness in the early postpartum period than at any other time in their lives. Almost a quarter of women who died between six weeks and one year postnatally died from psychiatric disorders [1].

The MBRRACE 2015 report identified a number of key messages to improve the care of pregnant women with mental illnesses. The report outlines 'red flags' which require urgent senior psychiatric assessment and considerations for admission to a mother and baby unit (Figures 1 and 2) [1]. Also highlighted is the importance of including a comprehensive psychiatric history in order to understand the progression of behavior. Furthermore, it is advised that an identified individual should co-ordinate care, where multiple mental health teams are involved [1]. The enquiry also details a new theme of partner and family involvement. This is especially important when a collateral history is required, particularly when capacity may be compromised. The MBRRACE enquiry also reports that some maternal deaths have been attributed to a failure in excluding organic causes, which may present as acute psychosis in pregnancy [1]. The case below is a successful model of how acute psychosis in pregnancy can be effectively managed in accordance to the recommendations of the MBRRACE 2015 report.

\section{Case report}

A multiparous 37-year-old woman, $36+5$ weeks pregnant, was found at home by her 7-year-old son, on her knees with blood on her hands. She presented to the emergency department with suicidal ideation, extreme distress and evidence of self-harm. She was deluded

- Recent significant change in mental state or emergence of new symptoms,

- New thoughts or acts of violent self-harm.

- New and persistent expressions of incompetency as a mother or estrangement from the infant.

Figure 1. Red flags requiring urgent psychiatric assessment [1]

- Rapidly changing mental state

- Suicidal ideation (particularly of a violent nature),

- Pervasive guilt or hopelessness

- Significant estrangement from the infant,

- New or persistent beliefs of inadequacy as a mother, evidence of psychosis.

Figure 2. Criteria for admission to a mother and baby unit [1] regarding the safety of her children, including her unborn baby, stating that 'they were all dead'. A collateral history taken from her husband revealed a three-week deterioration in her mood and that she had begun to reference films and books as part of her reality.

Her past medical history included: a congenital heart murmur and gestational diabetes, effectively managed by diet control and regular midwife reviews. As per the NICE guidelines, a depression and anxiety screen was carried out during the booking visit, to identify early signs of mental health problems that may arise during pregnancy [2]. On admission, the Consultant Obstetrician took a comprehensive collateral history from the husband, which ruled out any previous psychiatric history.

A full examination was unable to be carried out due to her high levels of anxiety, but there was evidence of self-harm in the form of scratch marks on her stomach and an injury to the back of her head. She was not verbally responsive, but was continuously muttering 'just kill me now. Due to the severity of her presentation, the emergency psychiatric team was contacted immediately and reviewed the patient within the hour. A mental state examination revealed that the patient lacked capacity at the time. Following the psychiatric review, she was immediately commenced on olanzapine and lorazepam, and was transferred to the maternity ward with 1:1 registered mental nurse and ward staff care, allowing for continuous direct observations. Due to her potential suicide intentions, ligature risks were identified within the bay she was placed in. All staff were notified of the patient's high suicidal risk and necessary measures were carried to minimise any potential risk to herself and others.

Once the patient was stabilized, investigations were performed to rule out possible organic causes such as: infection, hepatic encephalopathy, lupus, hypoglycaemia, thyroid dysfunction, electrolyte imbalance, substance abuse, trauma, space occupying lesions and domestic violence. Investigations carried out included: blood tests, blood cultures and arterial blood gases, urine MCS and toxicology screen, lupus screen, liver ultrasound and CT head. The CT scan showed no abnormalities however, blood results showed deranged liver function and metabolic acidosis and antibiotics were commenced in accordance with trust guidelines. Routine questions regarding domestic violence were also documented and ruled out.

Correspondence to: Radhika Viswanatha, Obstetric and Gynaeoclogy Consultant, Epsom and St Helier University Hospitals NHS Trust, UK, E-mail: m1200409@ sgul.ac.uk, radhika.viswanatha@nhs.net

Received: November 05, 2017; Accepted: December 01, 2017; Published: December 05, 2017 
Once the psychotic symptoms were stabilized, there was no indication for immediate delivery. However, a category 2 caesarean section was performed within 24 hours, due to an abnormal CTG, which showed foetal distress. The baby remained in special care postbirth due to peripheral cyanosis and some chest recession; leading to the patient expressing feelings of indifference and reduced bonding with her child. After discussion with the consultant psychiatrist, she was maintained on olanzapine and commenced on promethazine postoperatively. The liver function tests and acidosis normalised after the delivery of the baby.

Over the following days, she reported increased sleeping difficulties, which correlated with the deterioration in her mental state. On day four postnatally, she expressed distressing persecutory delusions and visual hallucinations. A subsequent psychiatric liaison assessment concluded that she was high risk and unfit for discharge. An admission to the Mother and Baby Unit (MBU) was discussed with the patient and her husband. As the patient voluntarily agreed to admission, sectioning under the mental health act was not required [3].

\section{Discussion}

Acute Psychosis in pregnancy is an obstetric emergency, due to the potential risk of death to both mother and baby. The MBRRACE report identifies numerous cases, which have resulted in fatal consequences, some of which were attributed to poor management. The MBRRACE (2006-2008) enquiry reported a case of a woman who became increasingly anxious throughout her normal pregnancy and developed delusional beliefs [4]. Although her mental health deteriorated postnatally, at no point was psychiatric referral considered. A diagnosis of anxiety was made; however, the community mental health team declined her referral [4]. A few weeks later this patient took her life by violent means. This case is an example where simple presentation, such as anxiety, can mask fatal diagnoses, resulting in the poorest of outcomes [4]. This highlights the importance of vigilance when dealing with psychotic symptoms in pregnancy and having a lower threshold for referral.

The MBRRACE (2006-2008) enquiry also outlines a case whereby the misattribution of physical symptoms to psychiatric illness led to the preventable death of a new mother [4]. A woman, with a known diagnosis of an autoimmune disorder, and an unremarkable psychiatric history, presented during pregnancy with complaints of lethargy and malaise. Although a psychiatric nurse assessment showed that the patient had no evidence of depressive illness, she was diagnosed with depression. She continued to deteriorate throughout her pregnancy and developed postnatal acute confusional state, which was again attributed to depression. The patient died shortly afterwards. The report suggested that the patient's deteriorating physical and cognitive state was likely due to her underlying autoimmune condition [4]. This case highlights the importance of excluding all possible organic causes before making a psychiatric diagnosis. As this was our patient's first presentation of psychosis, high clinical suspicion warranted all the appropriate investigations to exclude organic causes.

Evidence supports Mother and Infant Bonding (MIB) in the form of skin-skin contact [5]. This was significantly reduced from birth due to multiple factors. For example, due to the baby's own health concerns, specialist care was required, away from the mother's bedside. Efforts were made by the maternity ward staff to encourage MIB with supervised interactions including feeding and changing the baby. The husband was also encouraged to assist in these daily activities and thereby, supporting the mother and creating a safe environment for mother and baby.
Due to her antipsychotic medication, she was advised to avoid breast-feeding, which further impeded on MIB. There is limited data on the safety of antipsychotic medication in pregnancy and the post-natal period, however the NICE guidelines state that there are no specific contraindications. However, guidelines state that a discussion should be had with patients regarding the risks and benefits of treatment. She was continued on antipsychotics and vigorously monitored by the psychiatric team, obstetric consultant and mental health nurse. However, due to the fluctuating nature of post-partum psychosis, she was referred and admitted to the mother and baby unit for close monitoring.

Furthermore, patients should be advised, that this may be the primary presentation of bipolar affective disorder; the patient and the family should be advised on how to identity 'red flag' symptoms. Similarly, the patient should be told that this episode of pregnancyrelated psychosis may re-present, requiring closer monitoring in subsequent pregnancies. Further follow- up was not possible with this patient after discharge from mother and baby unit, however the importance of continuous monitoring for these patients should not be over-looked. Additionally, in line with the NICE guidelines on suspected child abuse and neglect, child protection officer and social services were contacted, as other children were involved [6].

This interesting case shows how the NICE guidelines can be effectively applied in practice, along side adhering to the recommendations outlined in the MBBRACE 2015 report.

\section{References}

1. Cantwell R, Knight M, Oates M, Shakespeare J (2015) on behalf of the MBRRACEUK mental health chapter writing group. Lessons on maternal mental health. In: Knight M, Tuffnell D, Kenyon S, Shakespeare J, Gray R, Kurinczuk JJ (Eds.) on behalf of MBRRACE-UK. Saving Lives, Improving Mothers' Care - Surveillance of maternal deaths in the UK 2011-13 and lessons learned to inform maternity care from the UK and Ireland Confidential Enquiries into Maternal Deaths and Morbidity 2009-13. Oxford: National Perinatal Epidemiology Unit, University of Oxford. Pp: 22-41.

2. NICE (2017) Antenatal and postnatal mental health: clinical management and service guidance.

3. Department of Health (2015) Mental Health Act 1983: reference guide. (1st edn.) Norwich: TSO.

4. Cantwell R, Clutton-Brock T, Cooper G, Dawson A, Drife J, et al. (2011) Saving Mothers' Lives: Reviewing maternal deaths to make motherhood safer: 2006-2008. BJOG 118 Suppl 1: 1-203. [Crossref]

5. Cleveland, L., Hill, C., Pulse, W., DiCioccio, H., Field, T. and White-Traut, R. (2017). Systematic Review of Skin-to-Skin Care for Full-Term, Healthy Newborns. J Obstet Gynecol Neonatal Nurs, 46: 857-869. [Crossref]

6. NICE (2017) Child abuse and neglect. NICE guideline.

Copyright: (C2017 Jain E. This is an open-access article distributed under the terms of the Creative Commons Attribution License, which permits unrestricted use, distribution, and reproduction in any medium, provided the original author and source are credited. 